Instituto de Salud Pública. Universidad Andrés Bello. Santiago de Chile.

angeniero Comercial, M.A. en Estudios Internacionales y Ph.D. en Ciencia Política de la Universidad de Hamburgo, Alemania.

bMaster en Salud Pública de la Universidad de Johns Hopkins.

Conflictos de intereses: ninguno que declarar.

Recibido el 16 de mayo de 2014, aceptado el 22 de noviembre de 2014 .

Correspondencia a: Pedro Aravena Lavín Salvador Sanfuentes 2355,

Piso 4. Santiago de Chile. aravena.pedro@gmail.com.

Dr. Manuel Inostroza minostroza@unab.cl

\section{¿Salud Pública o Privada? Los factores más importantes al evaluar el sistema de salud en Chile}

\author{
PEDRO ARAVENA L. ${ }^{a}$, MANUEL INOSTROZA P. ${ }^{b}$
}

\section{Evaluation of public and private health care systems}

\begin{abstract}
Background: There is a great degree of dissatisfaction with the Chilean health care system. Aim: To investigate which are the most relevant perceived factors when the health care system is evaluated. Material and Methods: Analysis of a survey about the Chilean health care system carried out during 2011, 2012 and 2013, involving 2,801 respondents. Results: The response capacity of emergency systems was the main factor considered for the evaluation of public and private health care systems. Respondents who were affiliated to private insurance systems also took into consideration the quality of medical infrastructure. Conclusions: There are different factors considered when public or private health care systems are evaluated.
\end{abstract}

(Rev Med Chile 2015; 143: 244-251)

Key words: Delivery of health care; Public Health; Public Opinion; Chile.
L a salud es un derecho fundamental, plasmado en la Constitución Política de Chile, en la cual se asume que el Estado provee una atención igualitaria y libre a la población, al mismo tiempo que consagra un sistema de carácter mixto, es decir, público y privado.

Desde el punto de vista de la eficacia del Sistema de Salud Chileno, se distinguen discrepancias desde varios ángulos temáticos. Primero, discusiones políticas y gremiales con respecto a la implementación de las reformas de salud ${ }^{1}$. Segundo, falta de espacios para la participación e involucramiento ciudadano con respecto a las decisiones sectoriales y desafíos de salud pública ${ }^{2}$.

En Chile, según la encuesta de la Superintendencia de Salud (2011), la gran mayoría de sus habitantes pertenece al sistema de salud público -alrededor de $80 \%$ de la población- mientras que únicamente $20 \%$ cuenta con un sistema privado de salud ${ }^{3}$. Interesante es que, según esta encuesta, únicamente $38 \%$ de la población encuestada manifiesta estar satisfecha con la atención pública, mientras que $61 \%$ con la atención privada, lo que podría denominarse una crisis de descontento con el sistema de salud público. Más aún, si consideramos los datos de la encuesta LAPOP (Proyecto de Opinión Pública de América Latina 2011), únicamente $5,2 \%$ de la población en Chile considera "buena" la calidad del servicio de salud público, siendo extremadamente bajo en comparación con otros países de la región como, por ejemplo, Argentina $(37,7 \%)$ y Uruguay $(47,5 \%)^{4}$. Ciertamente, las diferencias -con respecto al servicio de salud público y privado en Chile- pueden ser una causa de la disparidad en términos comparativos.

Entonces, ¿qué factores son los más importantes en la evaluación del sistema de salud? Para responder esta pregunta es posible considerar, principalmente, dos métodos: $1^{\circ}$. Estudios de opinión pública (de representación nacional o regional); $2^{\circ}$. Estudios específicamente a pacientes quienes evalúan, a través de cuestionarios, la calidad del servicio hospitalario.

La opinión pública es un método por excelencia utilizado en políticas públicas. De hecho, es parte central dentro de las democracias represen- 
tativas puesto que muestra las percepciones que tiene la población respecto a sus expectativas e intereses públicos 5 . Por otra parte, se distinguen estudios relacionados con la salud pública toda vez que se desea conocer las apreciaciones, en distintas dimensiones, sobre temas concernientes a problemas gubernamentales por resolver. Por ejemplo, en el año 2006, se realizó una encuesta en Estados Unidos para dar a conocer los problemas de salud más importantes para el gobierno, citándose los costos de atención como la principal prioridad ${ }^{6}$. Ahora bien, con respecto a tal método son escasas las investigaciones, basadas en metodología de regresión estadística, que estudian las preferencias de los habitantes por los sistemas de salud público y privado (estudios de opinión pública).

Con respecto al segundo método, éste ha sido fuertemente utilizado -en diversos estudioscon el objetivo de averiguar las diferencias en la calidad del servicio en los hospitales privados y públicos $^{7-11}$. El método más utilizado para medir la calidad de los servicios hospitalarios proviene de la mercadotecnia y corresponde al cuestionario denominado "SERVQUAL". Éste es el método más utilizado dentro de aquellos que evalúan la calidad de los servicios como, por ejemplo: technical and functional quality mode ${ }^{12}$, ideal value model of service quality ${ }^{13}$, internal service quality mode ${ }^{14}$, evaluated performance and normed quality mode $l^{15}$.

Con respecto a este último método, se ha concluido que en Grecia los pacientes de los hospitales públicos se encuentran mayormente satisfechos con la idoneidad de los médicos y enfermeras, sin embargo, los hospitales privados proporcionan mejores instalaciones físicas y menores tiempos de espera $^{16}$. Se ha argumentado que en los hospitales públicos de Singapur hay una carencia en términos de fiabilidad, seguridad, capacidad de respuesta, empatía, accesibilidad, asequibilidad y en términos tangibles (infraestructura) ${ }^{17}$. En Sudáfrica se ha hecho un interesante hallazgo: en los hospitales privados la empatía de las enfermeras hacia los pacientes, la seguridad y aspectos tangibles tienen un impacto positivo en la satisfacción con el servicio ${ }^{18}$.

Ciertamente, como lo señalan autores ya bastantes años antes, se distinguen diferencias entre los hospitales públicos y privados lo que ha llevado a una competitividad entre ambos por no fracasar frente a problemas de eficiencia ${ }^{19-20}$. En consecuencia, los clientes juegan un papel trascendental en la supervivencia de las entidades públicas y privadas toda vez que tienen la capacidad de evaluar un servicio $^{21-22}$.

El objetivo de este estudio es investigar qué factores son percibidos como más importantes al evaluar el sistema de salud en Chile. Igualmente, explorar la percepción de calidad que tienen del sistema de salud los usuarios de prestaciones públicas y privadas. Esto nos permitirá dar a conocer las causas del descontento de la población por el sistema de salud en ambos sectores.

\section{Metodología}

Se realizó un estudio observacional, analítico de corte transversal. A través de múltiples análisis econométricos se estudió la variable dependiente: nota del sistema de salud en general, la cual corresponde a una variable de percepción de calidad. Para ello se utilizaron modelos de regresión logística binominal puesto que los datos corresponden a encuestas cuya operacionalización de las variables pueden ser binarias, es decir, 0 y $1^{23}$. Con esto fue posible estudiar la relación entre distintas causalidades de la aprobación del sistema de salud. En consecuencia, tal variable se operacionalizó de la siguiente forma: ¿Qué nota le pone usted al sistema chileno de salud, en general? Use la escala de 1 a 7 , donde 1 es "Muy Malo" y 7 es "Muy Bueno". Una nota de 5 a 7 es categorizada con 1 y de 1 a 4 con 0 .

Se realizaron cuatro modelos econométricos puesto que se quiso averiguar el impacto de variados factores causales en la percepción de calidad del sistema de salud en distintas dimensiones. La primera dimensión corresponde a la población general de Chile, la segunda a la apreciación únicamente de los que cuentan con un seguro de salud público (Fonasa) y, por último, los que tienen un seguro de salud privado (Isapre).

El instrumento de recolección de datos correspondió a la Encuesta sobre el Sistema de Salud Chileno, del Instituto de Salud Pública de la Universidad Andrés Bello. Se realizó un corte transversal considerando los años 2011, 2012 y 2013 puesto que aquí se realizaron un mismo grupo de preguntas. Por consiguiente, se infirió que al abarcar un mayor número de datos se aportarían resultados más robustos. Se consideró una muestra representativa de la población de la Región Metropolitana y de Valparaíso, con una totalidad de 2.801 casos segmentados según 
grupo socioeconómico, sexo y región. El tamaño de muestra se asoció a un error estadístico de 5\% en caso de muestreo probabilístico y un nivel de confianza de $95 \%$.

\section{Resultados}

Al examinar los resultados descriptivos, lo primero que es posible distinguir es el alto descontento con el sistema de salud en general y que la mayor parte de la población pertenece a Fonasa (sistema de salud público). Llama la atención que el mayor disgusto es con respecto a la atención de urgencia, es decir, únicamente $30,3 \%$ señala que la atención al Servicio de Urgencia es oportuna. Otro factor de importancia, aunque menos crítico, es que únicamente $45,5 \%$ de la población indica que se sienten protegidos financieramente con su actual plan de salud. Por lo demás, las notas a los médicos es, en general, alta, al igual que la percepción del trato administrativo y de la calidad de la infraestructura médica. Por otro lado, se distingue que hay una predominancia de mujeres pertenecientes a Fonasa y, en cuanto a regiones, en Valparaíso es bastante mayor el número de afiliados, a tal organismo público, que en la Región Metropolitana (Tabla 1).

Ahora bien: ¿Qué factores son los más influyentes al momento de evaluar el sistema de salud? La tabla de correlaciones bivariadas muestra que la protección financiera y la atención de Servicios de Urgencias serían las variables que más se relacionan con la percepción del sistema de salud en general de Chile. Aquello, nos aproxima a posibles causalidades que se medirán en los modelos de regresión (Tabla 2).

El modelo multivariado de regresión logística binominal (Tabla 3 ) predice lo siguiente:

$1^{\circ}$ Hay una probabilidad aproximada de $63 \%$ de que la población adscrita a Fonasa estime que el sistema de salud en general es bueno, no ocurriendo lo mismo en el caso de los afiliados al sistema Isapre.

$2^{\circ}$ Hay entre $64 \%$ y $68 \%$ de probabilidad que la población que califica positivamente la atención médica, administrativa y la calidad de la infraestructura hospitalaria, también llegue a calificar positivamente el sistema de salud en general.

$3^{\circ}$ Es 2,2 veces más probable que un individuo indique que las atenciones de urgencia son opor- tunas y califique el sistema de salud con una buena nota, a que no lo haga. Tal resultado nos propone que el tiempo de respuesta de los Servicios de Urgencia es el factor más importante al momento de evaluar la percepción del sistema de salud en general $(\operatorname{Exp}(B)=2,215)$.

$4^{\circ}$ Es 2 veces más probable que un individuo señale sentirse protegido con su actual sistema de salud y, a su vez, evalúe positivamente el sistema de salud en general a que no llegue a suceder tal acontecimiento (Exp $(B)=2,096$. Aquel es el segundo factor en nivel de importancia, en el modelo que estudia la percepción de la totalidad de la población, con respecto al sistema de salud en general.

Se ha incluido la variable "hombres" pero no mujeres puesto que considerar ambas variables en el modelo habría sido redundante. Por igual motivo, los grados de libertad se han reducido con respecto a la variable "edad", es decir, se ha excluido la edad entre 18 y 39 años.

En la Tabla 4 es posible distinguir que los pertenecientes a Fonasa y al grupo socioeconómico $\mathrm{C} 2$, son quienes se sienten mayormente satisfechos con el sistema de salud en general. Es decir, la clase media-media.

En la Tabla 5 se observa que el segmento de la población afiliada a Fonasa evalúa el sistema de salud en general, primeramente, en base a los tiempos de espera en las atenciones médicas de urgencia $y$, en segundo lugar, los montos cancelados en la última atención.

La Tabla 6 muestra que el segmento de la población afiliada a Isapre evalúa el sistema de salud en general, primeramente, por la calidad de la infraestructura médica y, en segundo lugar, por su protección financiera.

\section{Discusión}

Estos resultados sugieren que la población evalúa el sistema de salud en general, primeramente, por la prontitud de las atenciones de urgencias. Este resultado es consistente con la evaluación que hacen los afiliados a Fonasa puesto que la variable "Atención Servicio de Urgencia" posee el mayor coeficiente de regresión. La segunda variable de mayor importancia, en tal caso, son los montos cancelados en la última atención médica. Por su parte, en el caso de los afiliados a Isapre, ellos 
Los factores más importantes al evaluar el sistema de salud en Chile - P. Aravena et al

Tabla 1. Frecuencia de las variables. $\mathbf{N}=\mathbf{2 . 8 0 1}$

\begin{tabular}{|c|c|c|c|c|c|c|}
\hline & & $\%$ & Sexo & $\begin{array}{c}\% \text { total } \\
\text { por sexo }\end{array}$ & Región & $\begin{array}{c}\% \text { total } \\
\text { por región }\end{array}$ \\
\hline \multirow[t]{2}{*}{ Nota al sistema de salud } & Bueno & $32,50 \%$ & Mujer & $\begin{array}{l}31,30 \% \\
34,00 \%\end{array}$ & $\begin{array}{l}\text { RM } \\
\text { Valnaraíso }\end{array}$ & $\begin{array}{l}29,90 \% \\
38,50 \%\end{array}$ \\
\hline & Malo & $67,50 \%$ & $\begin{array}{l}\text { Mujer } \\
\text { Hombre }\end{array}$ & $\begin{array}{l}68,70 \% \\
66,00 \%\end{array}$ & $\begin{array}{l}\text { RM } \\
\text { Valparaíso }\end{array}$ & $\begin{array}{l}70,10 \% \\
61,50 \%\end{array}$ \\
\hline Sexo & $\begin{array}{l}\text { Hombre } \\
\text { Mujer }\end{array}$ & $\begin{array}{l}46,70 \% \\
53,30 \%\end{array}$ & & & & \\
\hline Edad (años) & $\begin{array}{l}18-39 \\
40-59 \\
60-100\end{array}$ & $\begin{array}{l}38,20 \% \\
36,10 \% \\
25,70 \%\end{array}$ & & & & \\
\hline \multirow[t]{2}{*}{ Fonasa } & Sí & $68,20 \%$ & $\begin{array}{l}\text { Mujer } \\
\text { Hombre }\end{array}$ & $\begin{array}{l}71,70 \% \\
64,10 \%\end{array}$ & $\begin{array}{l}\text { RM } \\
\text { Valparaíso }\end{array}$ & $\begin{array}{l}65,90 \% \\
76,20 \%\end{array}$ \\
\hline & No & $31,80 \%$ & $\begin{array}{l}\text { Mujer } \\
\text { Hombre }\end{array}$ & $\begin{array}{l}28,30 \% \\
35,90 \%\end{array}$ & $\begin{array}{l}\text { RM } \\
\text { Valparaíso }\end{array}$ & $\begin{array}{l}34,10 \% \\
23,80 \%\end{array}$ \\
\hline \multirow[t]{2}{*}{ Isapre } & Sí & $20,90 \%$ & $\begin{array}{l}\text { Mujer } \\
\text { Hombre }\end{array}$ & $\begin{array}{l}18,80 \% \\
23,30 \%\end{array}$ & $\begin{array}{l}\text { RM } \\
\text { Valparaíso }\end{array}$ & $\begin{array}{l}22,60 \% \\
16,10 \%\end{array}$ \\
\hline & No & $79,10 \%$ & $\begin{array}{l}\text { Mujer } \\
\text { Hombre }\end{array}$ & $\begin{array}{l}81,20 \% \\
76,80 \%\end{array}$ & $\begin{array}{l}\text { RM } \\
\text { Valparaíso }\end{array}$ & $\begin{array}{l}77,40 \% \\
83,90 \%\end{array}$ \\
\hline \multirow[t]{2}{*}{$\begin{array}{l}\text { Gasto personal en salud } \\
\text { incluyendo medicamentos }\end{array}$} & Aumentado & $55,40 \%$ & $\begin{array}{l}\text { Mujer } \\
\text { Hombre }\end{array}$ & $\begin{array}{l}57,80 \% \\
52,80 \%\end{array}$ & $\begin{array}{l}\text { RM } \\
\text { Valparaíso }\end{array}$ & $\begin{array}{l}55,30 \% \\
52,00 \%\end{array}$ \\
\hline & Mantiene/Disminuido & $44,60 \%$ & $\begin{array}{l}\text { Mujer } \\
\text { Hombre }\end{array}$ & $\begin{array}{l}42,20 \% \\
47,20 \%\end{array}$ & $\begin{array}{l}\text { RM } \\
\text { Valparaíso }\end{array}$ & $\begin{array}{l}44,70 \% \\
48,00 \%\end{array}$ \\
\hline \multirow[t]{2}{*}{ Nota a médicos } & Buena & $74,80 \%$ & $\begin{array}{l}\text { Mujer } \\
\text { Hombre }\end{array}$ & $\begin{array}{l}76,10 \% \\
73,30 \%\end{array}$ & $\begin{array}{l}\text { RM } \\
\text { Valparaíso }\end{array}$ & $\begin{array}{l}74,90 \% \\
75,00 \%\end{array}$ \\
\hline & Mala & $25,20 \%$ & $\begin{array}{l}\text { Mujer } \\
\text { Hombre }\end{array}$ & $\begin{array}{l}23,90 \% \\
26,70 \%\end{array}$ & $\begin{array}{l}\text { RM } \\
\text { Valparaíso }\end{array}$ & $\begin{array}{l}25,10 \% \\
25,00 \%\end{array}$ \\
\hline \multirow[t]{2}{*}{ Trato personal administrativo } & Bueno & $64,60 \%$ & $\begin{array}{l}\text { Mujer } \\
\text { Hombre }\end{array}$ & $\begin{array}{l}65,20 \% \\
63,80 \%\end{array}$ & $\begin{array}{l}\text { RM } \\
\text { Valparaíso }\end{array}$ & $\begin{array}{l}61,90 \% \\
69,00 \%\end{array}$ \\
\hline & Malo & $35,40 \%$ & $\begin{array}{l}\text { Mujer } \\
\text { Hombre }\end{array}$ & $\begin{array}{l}34,80 \% \\
36,20 \%\end{array}$ & $\begin{array}{l}\text { RM } \\
\text { Valparaíso }\end{array}$ & $\begin{array}{l}38,10 \% \\
31,00 \%\end{array}$ \\
\hline \multirow[t]{2}{*}{ Infraestructura médica } & Buena & $68,40 \%$ & $\begin{array}{l}\text { Mujer } \\
\text { Hombre }\end{array}$ & $\begin{array}{l}71,10 \% \\
65,30 \%\end{array}$ & $\begin{array}{l}\text { RM } \\
\text { Valparaíso }\end{array}$ & $\begin{array}{l}66,10 \% \\
66,10 \%\end{array}$ \\
\hline & Mala & $31,60 \%$ & $\begin{array}{l}\text { Mujer } \\
\text { Hombre }\end{array}$ & $\begin{array}{l}28,90 \% \\
34,70 \%\end{array}$ & $\begin{array}{l}\text { RM } \\
\text { Valparaíso }\end{array}$ & $\begin{array}{l}33,90 \% \\
33,90 \%\end{array}$ \\
\hline \multirow[t]{2}{*}{ Protección financiera } & Protegida & $45,50 \%$ & $\begin{array}{l}\text { Mujer } \\
\text { Hombre }\end{array}$ & $\begin{array}{l}44,70 \% \\
46,30 \%\end{array}$ & $\begin{array}{l}\text { RM } \\
\text { Valparaíso }\end{array}$ & $\begin{array}{l}42,90 \% \\
49,50 \%\end{array}$ \\
\hline & No protegida & $54,50 \%$ & $\begin{array}{l}\text { Mujer } \\
\text { Hombre }\end{array}$ & $\begin{array}{l}55,30 \% \\
53,70 \%\end{array}$ & $\begin{array}{l}\text { RM } \\
\text { Valparaíso }\end{array}$ & $\begin{array}{l}57,10 \% \\
50,50 \%\end{array}$ \\
\hline \multirow[t]{2}{*}{ Atención servicio de urgencia } & Oportuna & $30,30 \%$ & $\begin{array}{l}\text { Mujer } \\
\text { Hombre }\end{array}$ & $\begin{array}{l}29,30 \% \\
31,50 \%\end{array}$ & $\begin{array}{l}\text { RM } \\
\text { Valparaíso }\end{array}$ & $\begin{array}{l}27,50 \% \\
37,90 \%\end{array}$ \\
\hline & Impuntual & $69,70 \%$ & $\begin{array}{l}\text { Mujer } \\
\text { Hombre }\end{array}$ & $\begin{array}{l}70,70 \% \\
68,50 \%\end{array}$ & $\begin{array}{l}\text { RM } \\
\text { Valparaíso }\end{array}$ & $\begin{array}{l}72,50 \% \\
62,10 \%\end{array}$ \\
\hline \multirow[t]{2}{*}{$\begin{array}{l}\text { Monto a cancelar en la última } \\
\text { atención }\end{array}$} & Bueno & $61,10 \%$ & $\begin{array}{l}\text { Mujer } \\
\text { Hombre }\end{array}$ & $\begin{array}{l}63,50 \% \\
58,40 \%\end{array}$ & $\begin{array}{l}\text { RM } \\
\text { Valparaíso }\end{array}$ & $\begin{array}{l}56,90 \% \\
67,30 \%\end{array}$ \\
\hline & Malo & $38,90 \%$ & $\begin{array}{l}\text { Mujer } \\
\text { Hombre }\end{array}$ & $\begin{array}{l}36,50 \% \\
41,60 \%\end{array}$ & $\begin{array}{l}\text { RM } \\
\text { Valparaíso }\end{array}$ & $\begin{array}{l}43,10 \% \\
32,70 \%\end{array}$ \\
\hline
\end{tabular}

Fuente: Elaboración propia en base a datos proporcionados por la encuesta del Instituto de Salud Pública de la UNAB. 
Tabla 2. Correlaciones

\begin{tabular}{|lc|}
\hline Sexo $(\mathrm{H})$ & $\begin{array}{c}\text { Nota al sistema } \\
\text { de salud }\end{array}$ \\
\hline Sexo $(\mathrm{M})$ & 0,029 \\
\hline Edad (años) $18-39$ & $-0,029$ \\
\hline Edad (años) $40-59$ & $-0,037^{*}$ \\
\hline Edad (años) $60-100$ & $-0,069^{* *}$ \\
\hline Fonasa & $0,117^{* *}$ \\
\hline Fonasa C1 & $0,078^{* *}$ \\
\hline Fonasa C2 & $-0,008$ \\
\hline Fonasa C3 & $0,089^{* *}$ \\
\hline Fonasa D & 0,005 \\
\hline Isapre & 0,017 \\
Gasto personal en salud incluyendo & $-0,068^{* *}$ \\
medicamentos & $-0,113^{* *}$ \\
\hline Nota a médicos & $0,210^{* *}$ \\
\hline Trato personal administrativo & $0,253^{* *}$ \\
\hline Infraestructura médica & $0,231^{* *}$ \\
\hline Protección financiera & $0,268^{* *}$ \\
\hline Atención servicio de urgencia & $0,263^{* *}$ \\
Monto a cancelar en la última atención & $0,191^{* *}$ \\
\hline $\mathrm{n}$ & 2.801 \\
\hline
\end{tabular}

* La correlación es significativa al nivel 0,05. ** La correlación es significativa al nivel 0,01. Fuente: Elaboración propia en base a datos proporcionados por la encuesta del Instituto de Salud Pública de la UNAB. evalúan el sistema de salud en general, primeramente, por la calidad de la infraestructura médica pasando a segundo lugar la protección financiera del plan de salud. Estos resultados podrían ser útiles al momento de definir -en cada uno de los subsistemas- políticas públicas que aborden estos elementos, es decir, las evaluaciones y percepciones que los beneficiarios tienen al estar en Isapres o Fonasa. Ambos segmentos de la población valoran de manera distinta su seguro y sus respectivas redes de prestadores de salud. Es decir, considerando las ventajas y deficiencias que ofrecen tanto el sistema de salud público como privado, nos acercamos a las dimensiones que se deben equilibrar para mantener satisfechos a sus afiliados. En el caso de la salud privada: infraestructura hospitalaria versus protección financiera en el copago de las prestaciones. En el caso de la salud pública: prontitud de la atención de urgencia versus montos en el copago de las prestaciones médicas.

Planteamos que todo lo que contribuya a mejorar la gestión clínica y asistencial de los prestadores públicos -produciendo una mejor asignación de recursos para que los pacientes gocen de una atención oportuna en los servicios de urgencia junto con bajas en los montos de copago de las prestaciones médicas- podría mejorar los niveles de satisfacción de los beneficiarios del Fonasa.

Tabla 3. Nota al sistema de salud chileno, muy bueno y bueno

\begin{tabular}{|lcccc|}
\hline & B & E.T. & Sig. & Exp (B) \\
Sexo (H) & 0,2188 & 0,0920 & 0,017 & 1,245 \\
Edad (años) 40-59 & $-0,1514$ & 0,1087 & 0,163 & 0,859 \\
Edad (años) 60-100 & 0,3449 & 0,1154 & 0,003 & 1,412 \\
Fonasa & 0,5308 & 0,1524 & 0,000 & 1,700 \\
Isapre & $-0,3899$ & 0,1768 & 0,027 & 0,677 \\
Gasto personal en salud incluyendo medicamentos & $-0,2423$ & 0,0924 & 0,009 & 0,785 \\
Nota a médicos & 0,4542 & 0,1332 & 0,001 & 1,575 \\
Trato personal administrativo & 0,5850 & 0,1173 & 0,000 & 1,795 \\
Infraestructura médica & 0,7377 & 0,1182 & 0,000 & 2,091 \\
Protección financiera & 0,7399 & 0,0989 & 0,000 & 2,096 \\
Atención servicio de urgencia & 0,7951 & 0,0960 & 0,000 & 2,215 \\
Monto a cancelar en la última atención & 0,3121 & 0,1027 & 0,002 & 1,366 \\
Constante & $-3,1972$ & 0,2270 & 0,000 & 0,041 \\
n & & & 2.801 & 0,186 \\
R2 Cox Snell & & & 0,259 & \\
R2 Negelkerke & & & 0 \\
\hline
\end{tabular}

Fuente: Elaboración propia en base a datos proporcionados por la encuesta del Instituto de Salud Pública de la UNAB. 
Los factores más importantes al evaluar el sistema de salud en Chile - P. Aravena et al

Tabla 4. Nota al sistema de salud chileno, muy bueno y bueno (la variable "Fonasa" se ha fraccionado según grupo socioeconómico)

\begin{tabular}{|lcccc|}
\hline & B & E.T. & Sig. & Exp (B) \\
Sexo (H) & 0,2028 & 0,0919 & 0,027 & 1,225 \\
Edad (años) 40-59 & $-0,1557$ & 0,1086 & 0,152 & 0,856 \\
Edad (años) 60-100 & 0,3150 & 0,1151 & 0,006 & 1,370 \\
Fonasa C1 & $-0,3066$ & 0,3005 & 0,308 & 0,736 \\
Fonasa C2 & 0,3415 & 0,1499 & 0,023 & 1,407 \\
Fonasa C3 & $-0,0173$ & 0,1147 & 0,880 & 0,983 \\
Isapre & $-0,7991$ & 0,1317 & 0,000 & 0,450 \\
Gasto personal en salud incluyendo medicamentos & $-0,2372$ & 0,0923 & 0,010 & 0,789 \\
Nota a médicos & 0,4469 & 0,1335 & 0,001 & 1,563 \\
Trato personal administrativo & 0,5678 & 0,1172 & 0,000 & 1,764 \\
Infraestructura médica & 0,7088 & 0,1184 & 0,000 & 2,031 \\
Protección financiera & 0,7223 & 0,0987 & 0,000 & 2,059 \\
Atención servicio de urgencia & 0,8108 & 0,0958 & 0,000 & 2,250 \\
Monto a cancelar en la última atención & 0,3171 & 0,1027 & 0,002 & 1,373 \\
Constante & $-2,7208$ & 0,1787 & 0,000 \\
n & & & 2.801 & 0,066 \\
R2 Cox Snell & & 0,184 & 0,257 & \\
R2 Negelkerke & & & \\
\hline
\end{tabular}

Fuente: Elaboración propia en base a datos proporcionados por la encuesta del Instituto de Salud Pública de la UNAB.

Tabla 5. Población con FONASA

\begin{tabular}{|c|c|c|c|c|}
\hline & \multicolumn{4}{|c|}{ FONASA } \\
\hline & B & E.T. & Sig. & $\operatorname{Exp}(B)$ \\
\hline Sexo $(H)$ & $-0,4072$ & 0,0867 & 0,000 & 0,666 \\
\hline Edad (años) 40-59 & 0,1670 & 0,0998 & 0,094 & 1,182 \\
\hline Edad (años) 60-100 & 0,3496 & 0,1113 & 0,002 & 1,419 \\
\hline Gasto personal en salud incluyendo medicamentos & $-0,1381$ & 0,0880 & 0,117 & 0,871 \\
\hline Nota a médicos & $-0,2351$ & 0,1197 & 0,049 & 0,790 \\
\hline Trato personal administrativo & $-0,3158$ & 0,1098 & 0,004 & 0,729 \\
\hline Infraestructura médica & $-0,7947$ & 0,1124 & 0,000 & 0,452 \\
\hline Protección financiera & $-0,7155$ & 0,0946 & 0,000 & 0,489 \\
\hline Atención servicio de urgencia & 0,4914 & 0,0983 & 0,000 & 1,635 \\
\hline Monto a cancelar en la última atención & 0,4596 & 0,0954 & 0,000 & 1,583 \\
\hline Constante & 1,7817 & 0,1470 & 0,000 & 5,940 \\
\hline$n$ & \multicolumn{4}{|c|}{2.801} \\
\hline R2 Cox Snell & \multicolumn{4}{|c|}{0,088} \\
\hline R2 Negelkerke & \multicolumn{4}{|c|}{0,123} \\
\hline
\end{tabular}

Fuente: Elaboración propia en base a datos proporcionados por la encuesta del Instituto de Salud Pública de la UNAB. 
Tabla 6. Población con ISAPRE

\begin{tabular}{|c|c|c|c|c|}
\hline & \multicolumn{4}{|c|}{ ISAPRE } \\
\hline & B & E.T. & Sig. & $\operatorname{Exp}(B)$ \\
\hline Sexo $(H)$ & 0,3396 & 0,1020 & 0,001 & 1,404 \\
\hline Edad (años) 40-59 & $-0,3996$ & 0,1145 & 0,000 & 0,671 \\
\hline Edad (años) 60-100 & $-1,0451$ & 0,1397 & 0,000 & 0,352 \\
\hline Gasto personal en salud incluyendo medicamentos & 0,3095 & 0,1042 & 0,003 & 1,363 \\
\hline Nota a médicos & 0,4727 & 0,1527 & 0,002 & 1,604 \\
\hline Trato personal administrativo & 0,2716 & 0,1326 & 0,041 & 1,312 \\
\hline Infraestructura médica & 1,2910 & 0,1526 & 0,000 & 3,636 \\
\hline Protección financiera & 1,0442 & 0,1127 & 0,000 & 2,841 \\
\hline Atención servicio de urgencia & $-0,6014$ & 0,1155 & 0,000 & 0,548 \\
\hline Monto a cancelar en la última atención & $-0,5662$ & 0,1111 & 0,000 & 0,568 \\
\hline Constante & $-2,9056$ & 0,1959 & 0,000 & 0,055 \\
\hline$n$ & \multicolumn{4}{|c|}{2.801} \\
\hline R2 Cox Snell & \multicolumn{4}{|c|}{0,129} \\
\hline R2 Negelkerke & \multicolumn{4}{|c|}{0,201} \\
\hline
\end{tabular}

Fuente: Elaboración propia en base a datos proporcionados por la encuesta del Instituto de Salud Pública de la UNAB.

En el caso del sector privado, para perseguir el mismo objetivo, junto con mantener o mejorar la protección financiera se debe continuar mejorando la calidad de la infraestructura médica. Aquello puesto que, según la metodología aquí propuesta, estos factores son relevantes para los beneficiarios de Isapre.

Cabe señalar que, pese a que la presente investigación recabó información relevante en torno a una muestra representativa de la población de las principales regiones del país, se presentaron algunas limitantes: los datos provienen de la opinión pública y no directamente de encuestas realizadas en los hospitales. Interesante sería contrastar tales resultados con un estudio a los propios pacientes dentro de los centros de atención públicos y privados. Es probable que los encuestados -al no estar en contacto con la calidad del servicio- puedan generar resultados distintos. Los estudios de esta índole suelen ser criticados por alteraciones en la percepción, toda vez que los encuestados son constantemente influenciados por los medios de comunicación, grupos de interés o líderes políti$\cos ^{24}$. Es por ello que se sugiere realizar estudios a través de cuestionarios SERVQUAL, cuando se desee comparar la calidad del servicio hospitalario público y privado desde el punto de vista de la percepción del cliente. El presente estudio busca ser un aporte en la discusión acerca de la calidad de los servicios en ambos casos y de las causas de la crisis de descontento en el sistema de salud chileno en general.

Anexo: Los datos de manejo de las variables pueden ser solicitados por los lectores interesados, al autor corresponsal.

\section{Referencias}

1. Instituto Salud y Futuro de la Universidad Andrés Bello. Salud del Bicentenario, Chile 2011-2020: Desafíos y Propuestas. 2011. Disponible en: http://www.ispandresbello. cl/wp-content/uploads/2013/10/salud-bicentenario.pdf [Consultado el 03 de septiembre de 2014].

2. Freile-Gutiérrez B. Participación ciudadana en el contexto de la evaluación de tecnologías sanitarias. Rev Med Chile 2014; 142 (1): 27-32.

3. Pezoa M. Estudio Opinión Usuarios del Sistema de Salud Conocimiento y Posicionamiento de la Superintendencia de Salud, GES-AUGE y otros aspectos de la Reforma 2011. Superintendencia de Salud y Adimark. Disponible en: http://www.supersalud.gob.cl/documentacion/569/ 
Los factores más importantes al evaluar el sistema de salud en Chile - P. Aravena et al

articles-6953_recurso_1.pdf [Consultado el 03 de abril de 2014].

4. Proyecto de Opinión Pública de América Latina (LAPOP). Sistema para Análisis de Datos en Línea 2011. Disponible en: http://lapop.ccp.ucr.ac.cr/Lapop.html [Consultado el 06 de abril de 2014].

5. Erikson R, MacKuen MB, Stimson JA. The Macro Polity. Cambridge: Cambridge University Press; 2002.

6. Kaiser Family Foundation Poll. Storrs, Conn: Roper Center for Public Opinion Research; 2006.

7. Donabedian A. Explorations in Quality Assessment and Monitoring. Ann Arbor MI: Health Administration Press; 1980.

8. Grönroos CA. Service Quality Model and Its Marketing Implications. European J Marketing 1984; 18 (4): 36-45.

9. Parasuraman A, Zeithaml V, Berry L. A Conceptual Model of Service Quality and Its Implications for Future Research. J Marketing 1985; 49 (4): 41-50.

10. Parasuraman A, Zeithaml V, Berry L. SERVQUAL: A Multiple Item-Scale for Consumer Perceptions of Service Quality. J Retailing 1988; 64 (1): 12-40.

11. Asubonteng P, McCleary KJ, Swan JE. SERVQUAL Revisited: A Critical Review of Service Quality. J Services Marketing 1996; 10 (6): 62-81.

12. Grönroos CA. Service Quality Model and Its Marketing Implications. European J Marketing 1984; 18 (4): 36-45.

13. Mattsson JA. Service Quality Model Based on an Ideal Value Standard. Internat J Service Industry Management 1992; 3 (3): 18-33.

14. Frost F. Mukesh K. INTSERVQUAL-An Internal Adaptation of the GAP Model in a Large Service Organization.
J Services Marketing 2000; 14 (5): 358-77.

15. Teas, KR. Expectations, Performance Evaluation, and Consumer`s Perceptions of Quality. J Marketing 1993; 57 (4): 18-34.

16. Angelopoulou P, Kangis P, Babis G. Private and Public Medicine: A Comparison of Quality Perceptions. Int. J. Health Care Qual. Assur 1998; 11 (1): 14-20.

17. Lim PC, Tang NK. A study of patient's Expectations and Satisfaction in Singapore Hospitals. Int. J. Health Care Qual. Assur 2000; 13 (7): 290-9.

18. Boshoff C, Gray B. The Relationship between Service Quality, Customer Satisfaction and Buying Intentions in the Private Hospital Industry. South Afr. J. Bus. Manage 2004; 35 (4): 27-37.

19. Parasuraman A, Zeithaml V, Berry L. A Conceptual Model of Service Quality and Its Implications for Future Research. J Marketing 1985; 49 (4): 41-50.

20. Zeithaml A, Parasuraman A, Berry L. Delivering Quality Service; Balancing Customer Perceptions and Expectations. Free Press 1990.

21. Reichheld F, Sasser WE. Zero defecting: Quality comes to Services. Harvard Buss Rev 1990; 68 (5): pp. 105-11.

22. Dawkins P, Reichheld F. Customer Retention as a Competitive Weapon. Directors Boards 1990; 14 (4): 42-7.

23. Klein R, Spady R. An Efficient Semi Parametric Estimator for Binary Response Models. Econometrica 1993; 61: 387-421.

24. Koch JW. Political Rhetoric and Political Persuasion: The Changing Structure of Citizen`s Preferences on Health Insurance During Policy Debate. Public Opinion Quarterly 1998; 62 (2): 209-29. 